\title{
Propuesta de Modelado Conceptual para definir, catalogar y seleccionar Patrones de Gobierno Electrónico
}

\section{Proposal of Conceptual Modelling to define, catalog and select Electronic Government Patterns}

Presentación: 06/10/2020

\section{Doctorando:}

\section{Oscar Carlos Medina}

Facultad Regional Córdoba, Universidad Tecnológica Nacional - Argentina

omedina@frc.utn.edu.ar

\section{Director: \\ Mario Alberto Groppo}

\section{Co-director:}

\section{Marcelo Martín Marciszack}

\section{Resumen}

En el desarrollo de la presente Tesis Doctoral "Definición de Patrones a partir de Buenas Prácticas para el desarrollo de sistemas de Gobierno Electrónico", se obtuvieron los siguientes resultados: a) un conjunto de tipos de Patrones que permiten definir Buenas Prácticas de e-Gob, b) un modelo de análisis para aplicación de Patrones de Buenas Prácticas en el Modelo Conceptual de sistemas de Gobierno Electrónico, y c) un método de catalogado y selección de estos Patrones para incorporar a un nuevo software público en la actividad de Modelado Conceptual. Este trabajo introduce en los conceptos claves de la investigación y expone un resumen descriptivo de los mencionados resultados, explicando el trayecto metodológico llevado a cabo para su elaboración.

Palabras clave: Patrones, e-Gob, Buenas Prácticas, Modelado Conceptual, Gobierno Electrónico.

\begin{abstract}
In the development of this PhD Thesis "Definition of Patterns based on Good Practices for the Electronic Government systems development", the following results were obtained: a) a set of types of Patterns that allow defining Good Practices e-Gob, b) an analysis model to applicate Good Practice Patterns in the Conceptual Model of Electronic Government systems, and c) a method of cataloguing and selection of these Patterns to incorporate into new public software in the Conceptual Modelling activity. This work introduces the key concepts of the research and presents a descriptive summary of the afore mentioned results, explaining the methodological path taken for its elaboration.
\end{abstract}

Keywords: Patterns, e-Gov, Good Practices, Conceptual Modelling, Electronic Government.

\section{Introducción}

Gobierno Electrónico es la aplicación de las TICs (Tecnologías de la Información y las Comunicaciones) en los procesos de Gobierno. Se considera a las TICs un "elemento dominante para mejorar factores urbanos como la calidad de vida, la operación urbana y los servicios, y su oferta económica, social y ambiental" (Estrada et al., 2018). 
También llamado e-Gobierno, o e-Gob (el prefijo "e" significa electrónico), abarca a los sistemas de información que dan soporte a los procesos de la Administración Pública.

Asociados a estos procesos se encuentran las Buenas Prácticas de Gobernanza. Se entiende por Buenas Prácticas de Gobernanza a "todas las iniciativas y experiencias que contribuyen a mejorar la efectividad de las actuaciones gubernamentales y que inciden positivamente en las condiciones de vida de los vecinos, logrando un impacto tangible en las comunidades"(Varela Rey, 2016). Las Buenas Prácticas de Gobernanza se utilizan como referencia en la construcción del software público (CLAD, 2015).

En pos de representar en forma estandarizada mejores prácticas, desde el enfoque de la Ingeniería de Software, los Patrones se destacan en la revisión bibliográfica del estado del arte. Un Patrón es un modelo que permite la reutilización de una solución exitosa para un mismo problema en distintos contextos. Las mencionadas Buenas Prácticas de e-Gob pueden ser descriptas empleando Patrones en la actividad de Modelado Conceptual del software público. La propuesta consiste en caracterizar patrones de Buenas Prácticas de e-Gob que se apliquen en el Modelo Conceptual de los sitios web del sector público, para que facilite su construcción con mayor nivel de calidad. La dimensión que se intenta optimizar es la de reusabilidad de implementaciones exitosas de sistemas de Gobierno Electrónico de Argentina. Ante lo cual, se formuló como objetivo general de la Tesis Doctoral la definición de Patrones a partir de Buenas Prácticas que puedan incorporarse en el Modelo Conceptual de sistemas de Gobierno Electrónico de Argentina, en el marco de un modelo iberoamericano de software público. En este artículo se explica el diseño metodológico que guía a la investigación y sus principales resultados.

\section{Desarrollo}

El diseño metodológico de la Tesis Doctoral tiene un enfoque cualitativo con estudios exploratorios de Buenas Prácticas de Gobierno Electrónico y de Patrones dentro del marco teórico de la Ingeniería de Software. Se realiza un análisis descriptivo de los Patrones de Buenas Prácticas de e-Gob que puedan aplicarse en el Modelo Conceptual de software público de Argentina.

En primer lugar, se confeccionó un mapeo sistemático de la literatura y un trabajo de aproximación descriptiva con la revisión de distintos documentos científicos de bibliotecas digitales virtuales reconocidas a nivel académico (Medina et al., 2018a). Se realizó una búsqueda exhaustiva de los conceptos esenciales de las Buenas Prácticas de Gobierno Electrónico y de las distintas estructuras y clasificación de patrones.

Se evaluaron las características básicas de las Buenas Prácticas gubernamentales identificadas. Con ellas, se formalizó una heurística de asociación a cada problemática de Gobierno Electrónico. Se definieron conceptos esenciales y aspectos relacionados a la construcción de patrones que se emplean en el desarrollo de software, sobre todo en la etapa de elicitación de requerimientos, en la actividad de creación del Modelo Conceptual.

Se seleccionaron los tipos de patrones que permitan definir Buenas Prácticas de e-Gob (ver Figura 1).

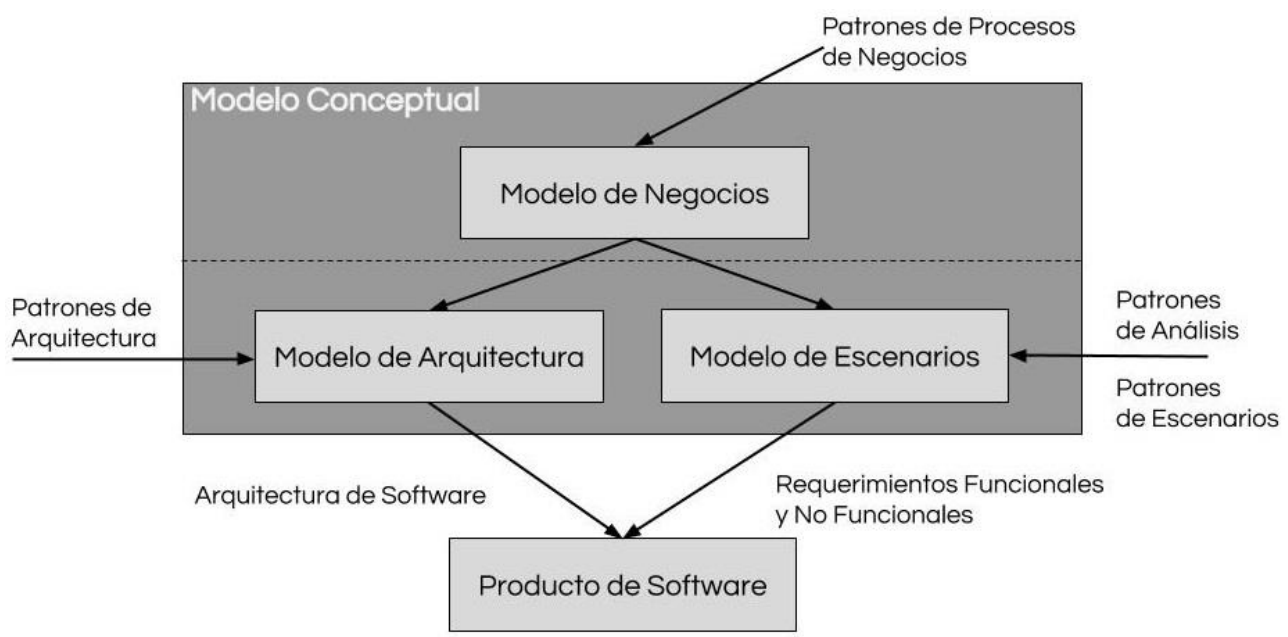

Figura 1: Patrones que permiten definir Buenas Prácticas de e-Gob en el Modelo Conceptual 
Se indagó la viabilidad de uso de estos patrones para el modelado de sistemas de Gobierno Electrónico y para verificar si la gestión de patrones contempla, y resuelve, los déficits de calidad identificados en el Modelado Conceptual de software público que no estuvo basado en Buenas Prácticas. Se desarrollaron distintos prototipos de sistemas públicos incorporando los patrones de Buenas Prácticas de e-Gob en la cantidad que sea necesaria para garantizar el resultado de la evaluación. Para la cual, se realizó una triangulación cualitativa al converger dos enfoques de la Ingeniería de Software, patrones y el Modelado Conceptual de sistemas de información.

Se elaboraron trabajos que fueron expuestos a consideración de la comunidad y publicados en revistas científicas y actas de congresos académicos, tanto a nivel nacional como internacional.

Se diseñó un experimento "off-line", sobre el nivel superior del Modelo Conceptual de un proyecto real de Gobierno Electrónico, que ya está implementado, con estudiantes y con el propósito específico de validar la hipótesis de la investigación. Se prevé su ejecución, si se autoriza en modalidad virtual, en el segundo semestre del corriente año.

\section{Resultados}

Con el fin de alcanzar el objetivo de la presente Tesis Doctoral, se obtuvieron los siguientes resultados:

a) un conjunto de tipos de Patrones que permiten definir Buenas Prácticas de e-Gob,

b) un modelo de análisis para aplicación de Patrones de Buenas Prácticas en el Modelo Conceptual de sistemas de Gobierno Electrónico,

c) un método de selección, catalogado e incorporación de estos Patrones a un nuevo software público en la actividad de Modelado Conceptual.

A continuación, se brindan más detalles de cada uno de estos resultados.

Patrones que permiten definir Buenas Prácticas de e-Gob. El concepto de patrón fue el formulado por el arquitecto Christopher Alexander quien afirma que "cada patrón es una regla de tres partes, que expresa una relación entre un cierto contexto, un problema y una solución" (Alexander, 1979). Esta idea de patrón es aplicada en Patrones de Diseño de software por Gamma, Helm, Johnson, y Vissides, acorde al paradigma de la programación orientada a objetos (Gamma et al., 1994). A partir de esta publicación se incorporaron patrones a las distintas fases del desarrollo de software creando tipos específicos para cada una de ellas. Del análisis del marco conceptual de los temas Buenas Prácticas de Gobierno Electrónico y Patrones, se pudo colegir que los tipos de patrones que permiten definir Buenas Prácticas de e-Gob en el Modelado Conceptual del software público son los siguientes: Patrones de Procesos de Negocios, Patrones de Análisis, Patrones de Escenario y Patrones de Arquitectura. Las capas del Modelo Conceptual en las que aplican cada uno de estos tipos de patrones se puede apreciar gráficamente en la Figura 1. Los Patrones de Procesos de Negocios poseen el mayor nivel de abstracción permitiendo trabajar con el modelado del negocio, posibilitando describir los recursos del modelo, organizaciones, objetivos, procesos y normas de la empresa (Eriksson y Penker, 2000). Los Patrones de Análisis y Patrones de Escenarios aplican sobre la capa de requerimientos funcionales y no funcionales del modelado. Los Patrones de análisis son grupos de conceptos que representan en conjunto un modelo de negocios relevante dentro de un dominio de aplicación y los Patrones de Escenarios son "descripciones narrativas de las interacciones entre los usuarios y los sistemas propuestos" (Potts, 1995). En cambio, los Patrones de Arquitectura forman parte de la arquitectura lógica de un sistema, lo que hace necesaria su definición en la fase de construcción, pero es factible y conveniente su inclusión en el Modelo Conceptual. Los Patrones de Arquitectura son un conjunto predefinido de subsistemas con sus responsabilidades y una serie de recomendaciones para organizar los distintos componentes (Buschmann et al., 1996).

Modelo de análisis para aplicación de Patrones de Buenas Prácticas en el Modelo Conceptual de sistemas de Gobierno Electrónico. El Modelo Conceptual de un sistema de información tiene como finalidad identificar y explicar los conceptos significativos en un dominio de problema, elicitando los atributos y las asociaciones existentes entre ellos. Según Sommerville el Modelo Conceptual del sistema se realiza en las primeras actividades del "Proceso Unificado Racional" de desarrollo de software: modelado de negocios, elicitación de requerimientos, análisis y diseño (Sommerville, 2011). Los Patrones que se aplican al Modelado Conceptual cumplen la función de reutilizar el 
conocimiento y experiencia de sistemas anteriores encapsulado en soluciones de análisis y diseño concretas, permitiendo la verificación y validación de los requerimientos funcionales. A partir del Modelado de Negocio es factible una derivación de la arquitectura de software que les da soporte en el marco de la metodología MDD (Model Driven Development), en español Diseño Dirigido por Modelos. El MDD utiliza modelos diagramáticos que se van transformando en las distintas etapas de construcción de un sistema. "MDD es la evolución natural de la ingeniería de software basada en modelos enriquecida mediante el agregado de transformaciones automáticas entre modelos" remarca Pons (Pons et al., 2010). El modelo de análisis que plantea este trabajo posibilita elaborar, clasificar, almacenar y seleccionar patrones de un conjunto acotado de patrones específicos de Gobierno Electrónico denominado catálogo. En el dominio del Modelado Conceptual nos referimos específicamente a Patrones de Negocios, Patrones de Análisis, Patrones de Escenarios y Patrones de Arquitectura. La primera instancia entonces, es definir y catalogar un nuevo patrón a partir de una experiencia exitosa de implementación de un sistema del sector público, por ejemplo, un nuevo Patrón de Negocios. De este tipo de patrones, los que adquieren mayor representatividad son los Patrones de Problema - Objetivo ya que explicitan la relación problema-solución de un proceso de negocio al cual dieron soporte. Al catalogar un Patrón de Problema - Objetivo, se almacena además para el mismo proceso de negocio los otros patrones que complementan la descripción funcional: los Patrones de Procesos y los Patrones de Recursos y Reglas. En cuanto a los Patrones de Negocios se pone a consideración una innovación con un gráfico unificado que permite una ágil visualización de los Patrones de Modelado de Procesos: Estructura básica de proceso, Proceso de interacción, Proceso de suministro en capas y Flujo de acción de trabajo (Marciszack et al. 2018). Además de la información de la "Plantilla de Patrones de Negocios" definida por Eriksson y Penker (Eriksson y Penker, 2000), se le agregan palabras claves que tiene una valorización o peso de relevancia acorde a la capacidad del patrón para resolver los problemas del proceso de negocio para el cual fue implementado. Este componente es necesario para desarrollar un tesauro estandarizado y específico para el catálogo y que explore con mayor eficiencia las funcionalidades de la minería de texto al analizar la descripción inicial de un problema en la búsqueda. Por tal motivo, elegir un patrón es otra de las tareas críticas que debe resolver toda actividad de modelado que use patrones. Para esta segunda instancia se pone a consideración un método que es desarrollado en el apartado siguiente. Por último, una vez seleccionado el patrón, o conjunto de patrones, más adecuados se incorpora a cada una de las capas del Modelado Conceptual como línea base del documento de Especificación de Requisitos de Software, ERS. El análisis de requisitos es la actividad más importante en el desarrollo de un proyecto software, ya que de un correcto análisis dependerá la correcta implementación de la aplicación. La ERS supone una especie de contrato entre usuario y diseñadores del software, en el que unos indican sus necesidades, mientras que los otros proponen soluciones para implementar lo que se indica en el documento (Sommerville, 2011). El empleo de patrones en el inicio del Modelado Conceptual aporta una serie de beneficios significativos en la construcción del sistema, que fueron observados en los primeros casos de estudio. Cada resultado parcial obtenido en el proceso de conformación de este modelo de análisis, fue presentado y publicado como artículo en actas, o capítulo de libro, de los siguientes congresos científicos: CISTI de España (Medina et al., 2018c), CIMPS de México (Medina et al., 2018d) (Meloni et al., 2019), ICITS de Ecuador (Medina et al., 2020), WICC, CONAIISI y la 5 5 Jornada de Doctorandos de Argentina (Medina et al., 2018b, 2018e, y 2018f).

Método de catalogado y selección de Patrones para incorporar en el Modelado Conceptual de un software público. Definir un nuevo patrón, y elegir uno preexistente de un conjunto acotado denominado catálogo, son actividades fundamentales que debe resolver todo modelo de análisis que use patrones. Para validar de forma experimental el modelo de análisis de esta investigación, se elaboró una propuesta para catalogar y seleccionar Patrones de Negocios para el Modelado Conceptual de sistemas, y se implementa con un prototipo denominado "PatCat", Pattern Catalogue. Esta aplicación web propia, en su versión inicial, permite la gestión de un catálogo de Patrones de Negocios aplicados en casos reales de e-Gob para que facilite su construcción simplificando y brindando mayor claridad en la fase de modelado. Este método, parte del análisis del lenguaje natural en la descripción del problema por parte del diseñador. Para lo cual se utilizan técnicas de minería de texto que permitan el análisis de componentes en patrones del catálogo, e interactuando con el diseñador, elaboren una recomendación del patrón más adecuado al problema descripto. Implementado en un catálogo de Patrones de Diseño, Bouassida presenta un método interactivo de selección de estos patrones (Bouassida et al., 2015). También Paz Menvielle plantea la búsqueda de patrones en escritura de texto libre, dentro de un dominio acotado, representado en una base de datos de grafos dirigidos (Paz Menvielle et al., 2018). La búsqueda de palabras claves identificadas en dicha descripción, se realiza en un primer momento en el modelo del negocio y se puede aplicar tempranamente en el Modelado Conceptual. Los pasos consisten en encontrar un Patrón de Objetivos del sistema que se está modelando y que tengan asociadas las palabras claves, ya que en este tipo de patrón se describen los problemas que resuelve el proceso. Luego se obtiene el Patrón de Procesos de Negocios en un gráfico unificado, planteado en (Marciszack et al., 2018), que desarrolla el 
modo en que se relacionan todos los procesos intervinientes y cómo intercambian recursos entre ellos. La especificación del proceso principal de este patrón puede tener otros tipos de patrones asociados, como por ejemplo Patrones de Análisis y Patrones de Escenarios, que complementan la especificación funcional de requisitos de software.

\section{Conclusiones}

Se considera que una propuesta de Modelado Conceptual para sistemas de Gobierno Electrónico, como la descrita en el presente trabajo, pone a disposición de la industria de software local, no solo de un catálogo de Buenas Prácticas en lo que se refiere fundamentalmente a Patrones e-Gob, sino también de una estrategia que facilite la reusabilidad asegurando la calidad del software desde etapas tempranas de su modelado a través del uso de patrones. A su vez, se garantiza la utilización de Buenas Prácticas de gobernanza y un desempeño más eficiente de soluciones que contemplan de forma clara y completa, posibles requisitos necesarios para etapas posteriores del ciclo de desarrollo de sistemas de información gubernamentales.

Las áreas de informática estatales, consultoras de software privadas y las Universidades existentes en nuestro medio, también tendrán la posibilidad de acceder a los resultados de esta Tesis Doctoral. Por lo que se prevé que el desarrollo colaborativo y abierto de un catálogo de patrones de Buenas Prácticas de Gobierno Electrónico, coadyuvará a mejores servicios digitales en las diversas organizaciones públicas de nuestro país, contribuyendo también a una mejora en el desarrollo productivo del software nacional.

Para finalizar, las próximas actividades planificadas son la ejecución del experimento, análisis y difusión de sus resultados, en cuanto a la aplicación de patrones de Buenas Prácticas en el Modelado Conceptual de sistemas de gobierno digital, Dichas conclusiones se presentarán en congresos y publicaciones científicas y se volcarán en la redacción definitiva de la Tesis Doctoral.

\section{Referencias}

Alexander, C. (1979). The Timeless Way of Building. Oxford University Press - New York.

Bouassida, N., Jamoussi, S., Msaed, A., Hanêne Ben-Abdallah, H. (2015). An Interactive Design Pattern Selection Method. Publicado en actas de J.UCS (Journal of Universal Computer Science), 21, 13, 1746-1766.

Buschmann, F., Sommerlad, P., Stal, M., Meunier, R., Rohnert, H. (1996). Pattern-Oriented Software Architecture. A System of Patterns. Volume 1, Ed. Wiley.

CLAD Centro Latinoamericano de Administración para el Desarrollo (2015). Cartas Iberoamericanas del CLAD, Secretaría de la Función Pública de la República del Paraguay.

Eriksson, H.E. y Penker, M. (2000). Business Modeling with UML: Business Patterns at Work. OMG Press.

Estrada, E., Maciel, R., Peña Pérez Negrón, A., López Lara, G., Larios, V., Ochoa, A. (2018). Framework for the Analysis of Smart Cities Models, in Mejia, J. et al. (Ed.): Trends and Applications in Software Engineering. Proceedings of the 7th International Conference on Software Process Improvement (CIMPS 2018), 261-269, Springer.

Gamma, E., Helm, R., Johnson, R., Vlissides, J. (1994). Design Patterns - Elements of Reusable Object-Oriented Software. Addison Wesley, Pearson Education Limited.

Marciszack, M.M., Moreno, J.C., Sánchez, C.E., Medina, O.C., Delgado, A.F., Castro, C.S. (2018). Patrones en la construcción del Modelo Conceptual para sistemas de información. Editorial edUTecNe, U.T.N.

Medina, O.C, Marciszack, M.M., Groppo, M.A. (2018). Aproximación descriptiva a las Buenas Prácticas de Gobierno Electrónico y a su incorporación en el Modelado Conceptual de Sitios Web Públicos de Argentina. Revista

“Tecnología y Ciencia” en línea, №31, Artículo 107, Universidad Tecnológica Nacional, Argentina. 
Medina, O.C, Marciszack, M.M., Groppo, M.A. (2018). Un Modelo de Análisis para aplicación de patrones de Buenas Prácticas en el Modelado Conceptual de Gobierno Electrónico. Publicado en actas de WICC 2018, Red UNCI y UNNE Universidad Nacional del Nordeste.

Medina, O.C, Marciszack, M.M., Groppo, M.A. (2018). Proposal for the patterns definition based on good practices for the electronic government systems development. Publicado en actas de CISTI 2018 - 13th Iberian Conference on Information Systems and Technologies.

Medina, O.C., Pérez Cota, M., Marciszack, M.M., Martin, S.M., Pérez, N., Dean, D.D. (2018). Conceptual Modelling of a Mobile App for Occupational Safety Using Process and Objectives Patterns. Trends and Applications in Software Engineering. Publicado en actas de 7th International Conference on Software Process Improvement (CIMPS 2017), 186-195, Springer.

Medina, O.C, Cánepa, P.A., Gruppo, M.O., Groppo, M.A. (2018). Un caso de estudio de patrones de Gobierno Electrónico para gestión de consultas de ciudadanos. Publicado en actas de CONAIISI 2018, Red RIISIC, CONFEDI y Universidad CAECE.

Medina, O.C., Groppo, M.A., Marciszack, M.M. (2018). Una aproximación a la definición de Patrones a partir de Buenas Prácticas para el desarrollo de sistemas de Gobierno Electrónico. Publicado en actas de Jornadas y Eventos Académicos de UTN (AJEA), 4, 274-278, Universidad Tecnológica Nacional.

Medina, O.C., Romero, M.S., Romero, R.A., Martin, S.M., Marciszack, M.M. (2020). Using Architecture Patterns in the Conceptual Model of an eGov Software. Trends and Applications in Information Technology and Systems.

Proceedings of the 2020 International Conference on Information Technology \& Systems (ICITS 2020), 54-63, Springer.

Meloni, B.E., Pérez Cota, M., Medina, O.C., Marciszack, M.M. (2019). Objectives Patterns Applied to the Business Model of a Public Education System. Trends and Applications in Software Engineering. Proceedings of the 8th International Conference on Software Process Improvement (CIMPS 2019), 13-22, Springer.

Paz Menvielle, M.A., Corso, C.L., Guzmán, A., Casatti, M.G., Ligorria, K. (2018). Búsqueda de patrones en un dominio representado en una base de datos de grafos dirigidos. Publicado en actas de CONAIISI 2018, Red RIISIC, CONFEDI y Universidad CAECE.

Pons C., Giandini R., Pérez G. (2010). Desarrollo de Software dirigido por Modelos (Conceptos teóricos y su aplicación práctica), Edulp - Editorial de la Universidad de la Plata.

Potts, C. (1995). Using Schematic Scenarios to Understand User Needs. Publicado en actas de 1st Conference on Designing interactive systems: processes, practices, methods, \& techniques (DIS'95), 247-256. ACM.

Sommerville, I. (2011). Ingeniería de Software. 9a Edición en español, Pearson.

Varela Rey, A. (2016). Beneficios del intercambio de buenas prácticas municipales, INNOTEC Gestión, 7, 55-59, Laboratorio Tecnológico del Uruguay. 